\title{
Conservative Kinematic Alignment Strategies Report Higher Incidence of Mid-Term Pain Following TKA: A Retrospective Study
}

\author{
Edgar A Wakelin $\mathrm{PhD}^{1^{*}}$, Joshua G Twiggs $\mathrm{PhD}^{1}$, Brett Fritsch $\mathrm{MD}^{2}$, \\ Michael Solomon $\mathrm{MD}^{3}$, Brad P Miles $\mathrm{PhD}^{1}$
}

1360 Knee Systems, Sydney, Australia

${ }^{2}$ Sydney Orthopaedic Research Institute, Sydney, Australia

${ }^{3}$ Sydney Orthopaedics Specialists, Sydney, Australia

joshua@kneesystems.com, brad@_kneesystems.com

\begin{abstract}
Kinematic alignment (KA) aims to restore the pre-arthritic geometry of the joint and has been shown to produce similar or improved outcomes compared to mechanical alignment (MA). However, there are significant challenges when attempting to restore outlier joint anatomy. This study sought to investigate how achieved TKA alignment correlates with 12 month patient outcomes. The study considered three categories of achieved TKA alignments: KA, MA, or conservative KA (restricting coronal component alignments to within $5^{\circ}$ and combined coronal alignment to within $3^{\circ}$ of neutral). 369 patients were analysed; each patient had pre- and post-operative CT scans and a 12-month postoperative Knee Osteoarthritis \& Outcome Score (KOOS). From CT scans, component positions were measured, and patients were categorized to one of the three categories. The conservative KA group were least likely (76\%) to reach the threshold KOOS Pain score set by the Patient Acceptable State Score (PASS); the full KA group was most likely (95\%) to reach the threshold, whilst the MA group was in between these two groups (88\%). These results demonstrated an increase in pain when the achieved alignment formed a compromise between a restorative and a reconstructive approach, and further suggests that conservative approaches to kinematic alignment may require a high level of discretion.
\end{abstract}




\section{Introduction}

Kinematic alignment (KA) aims to restore the pre-arthritic geometry of the joint and has been shown to produce similar or improved outcomes compared to mechanical alignment (MA) (Courtney and Lee 2017). There are significant challenges however when attempting to restore outlier joint anatomy. Conservative KA approaches are a means of achieving a compromise between KA and MA philosophies when unusual or outlier anatomy is encountered (Almaawi et al. 2017). Unlike KA however, there have been no studies investigating the outcome of conservative KA compared to either MA or KA (Riviere et al. 2017). This study sought to investigate how achieved TKA alignment correlates with short term patient outcomes.

\section{Method}

A database of TKA patients operated on by nine surgeons from 1-Jan-2014 was accessed. All patients had a pre-operative CT scan segmented as part of routine surgical planning and a post-operative CT scan for post-operative analysis of the component positioning. The achieved alignment was measured from the post-operative CT scan and categorised as either KA, MA, or a conservative approach to KA according to the technique described by Almaawi et al. (Almaawi et al. 2017) restricting coronal component alignments to within $5^{\circ}$ and combined coronal alignment to within $3^{\circ}$ of neutral. Component positions were measured, and patients were binned in to one of three categories that represented the lowest combined angular error from the achieved alignment in 3 dimensions.

The relationship between achieved alignment and a 12-month postoperative Knee Osteoarthritis \& Outcome Score (KOOS) was determined. All TKAs were performed using CR or PS Omni Apex (Raynham) implants.

\section{Results}

A total of 369 TKA knees were identified in the database. $60 \%$ (221) were female, with an average age of 70.1 years (+/- 8.2). $21 \%$ (76) of the knees were classified as KA, with $10 \%$ of the total (37) being conservative KA rather than fully kinematically aligned.

When dichotomising patients into those with a KOOS Pain score satisfying the Patient Acceptable State (PASS) Score (less than or greater than 70)(Muller et al. 2013) a trend was found, in which $76 \%$ (28/37) of the conservative KA group, 88\% (257/293) of the MA group and 95\% (37/39) of the full KA group reached the PASS score. When combining the MA group with the full KA group and comparing the outcome with that of conservative KA, the difference was statistically significant $(p=0.026)$. Under these conditions, the conservative KA group reported a 1.8x greater risk of failing to reach the PASS KOOS pain threshold, see Error! Reference source not found. This relationship remained significant $(\mathrm{p}<0.05)$ upon further analysis to investigate only knees with anatomy which would invoke conservative KA rules. 


\section{Discussion}

MA has been the gold standard for implant survivorship and joint stability (Riviere et al. 2017). This is achieved by performing neutral coronal and axial resections and releasing soft tissue to generate a balanced knee. KA conversely, maintains the soft tissue envelope and modifies the bony resection to recreate the pre-osteoarthritic joint and maintain a natural soft tissue balance. Conservative KA is a compromise of these two philosophies in which component alignments may be placement more neutral to preserve joint stability, and soft tissue release may be used to balance the knee. Rather than achieving improved joint stability compared to KA, by matching the medio-lateral resections, and improved native soft tissue balance compared to MA, by reducing the number of releases (Dossett et al. 2014), the compromises inherent in conservative KA is reported here to increase the rate of post-operative pain. The origin of the increased rate of pain is currently unknown but may be due to the nature of the compromise, leading to instability in both the supporting soft tissue structures and articulating components. Nevertheless $74 \%$ of conservative KA knees achieved the PASS score, indicating ideal component placement may be patient specific. Routine pre-operative kinematic analysis with computational models represent a non-invasive solution to this and allow alignment strategies such as MA, KA or conservative KA to be selected on a multifactorial patient specific basis.

This study has several limitations. First the surgeon technique and target alignment were uncontrolled across nine surgeons, which may not be sufficient for generalisability. Second, all knees were assigned an alignment according to the best fit, without an assessment of how close the achieved alignment is compared to the ideal alignment category. Future work will investigate the origin of the increased rate of post-op pain to determine alignment strategies to optimise patient outcome.

\section{Conclusions}

This study showed an increase in pain when the achieved alignment formed a compromise between a restorative and a reconstructive approach. This suggests that conservative approaches to kinematic alignment that maximise patient outcome may require selective application of kinematic alignment rather than blanket application of modified anatomical rules. 


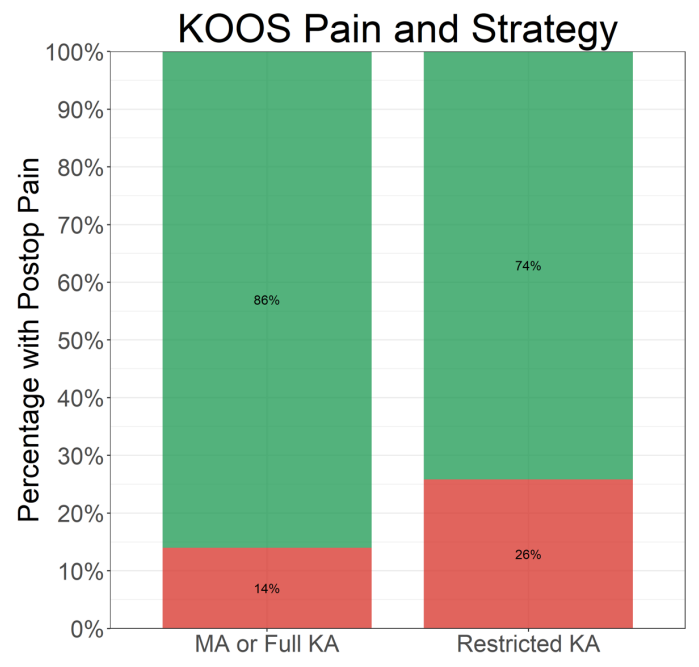

Figure 1: Comparison of rate of post-op pain (KOOS pain $<70)$ with respect to achieved alignment

\section{References}

Almaawi, A. M., J. R. B. Hutt, V. Masse, M. Lavigne, and P. A. Vendittoli. 2017. 'The Impact of Mechanical and Restricted Kinematic Alignment on Knee Anatomy in Total Knee Arthroplasty', $J$ Arthroplasty, 32: 2133-40.

Courtney, P. M., and G. C. Lee. 2017. 'Early Outcomes of Kinematic Alignment in Primary Total Knee Arthroplasty: A Meta-Analysis of the Literature', J Arthroplasty, 32: 2028-32 e1.

Dossett, HG, NA Estrada, GJ Swartz, GW LeFevre, and BG Kwasman. 2014. 'A randomised controlled trial of kinematically and mechanically aligned total knee replacements: two-year clinical results', The bone \& joint journal, 96: 907-13.

Muller, Bart, Mohammad A Yabroudi, Chung-Liang Lai, Andrew Lynch, Christopher D Harner, C Niek Van Dijk, Freddie H Fu, and James J Irrgang. 2013. 'Defining Patient Acceptable Symptom State Thresholds for the IKDC Subjective Knee Form and KOOS for Patients Undergoing ACL Reconstruction', Orthopaedic journal of sports medicine, 1: 2325967113S00069.

Riviere, C., F. Iranpour, E. Auvinet, S. Howell, P. A. Vendittoli, J. Cobb, and S. Parratte. 2017. 'Alignment options for total knee arthroplasty: A systematic review', Orthop Traumatol Surg Res, 103: 1047-56. 